\title{
Sensing as a Service Model for Pulse Oximeter Supported by Internet of Things
}

\author{
https://doi.org/10.3991/ijes.v5i3.6618 \\ Ms.Shruti Daddikar( $\left.{ }^{\bowtie}\right)$, Dr.Roshani Ade \\ Savitribai Phule Pune University, Pune, Maharashatra, India \\ Shruti.daddikar@gmail.com.
}

\begin{abstract}
Pulse oximetry is technique used for measurement of oxygen saturation of the blood. It is a simple method used for measurement of how well oxygen is being transported to different organs of your body from your heart. In present scenario pulse oximeter offers two parameters i.e. heart rate and oxygen saturation; however it is implemented only for clinical purpose. Pulse oximeters which compute $\mathrm{SpO}_{2}$ using transmittance photo plethysmography (PPG), is widely accepted for continuous monitoring. The purpose of this paper is an approach to recognize oxygen level in people's blood. Our method uses $\mathrm{SpO}_{2}$ sensor (Blood oxygen saturation level) and MSP432 microcontroller. By using $\mathrm{SpO}_{2}$ sensor to sense oxygen level from the blood and Beer's Lambert Laws to get normal oxygen level value and that oxygen level value display on LCD display. The proposed method requires less no of hardware so it reduces the production cost also.
\end{abstract}

Keywords-Pulse oximetry, $\mathrm{SpO}_{2}$ Sensor, MSP432 microcontroller, heartrate, oxygen saturation

\section{Introduction}

The pulse oximeter is a medical gadget that identifies the oxygen level in the blood. People need to continually check the oxygen level in the blood while they feel with illness. To identify the oxygen level in the blood is more important because some human body organs will be damaged when the oxygen level is not sufficient in the human body. It is more important to monitor oxygen levels because some human organs like kidney, liver will be permanently damaged because lack of oxygen. [1] People analyze the oxygen level. This section contains information on the pulse oximeter function principle and basic physiology information about blood oxygenation.

The sensor $\mathrm{SpO}_{2}$ is used for measuring oxygen in the blood. The pulse oximeter can find out oxygen level by using red light and infra-red light. a pulse oximeter comprises of two light sources and a photodetector associated with a signal processing unit.

Pulse oximeters have gained widespread acceptance in the medical community for several reasons. Since the first production of pulse oximeters in the early 1980s, pulse oximeters have been recognized and praised for their low cost and simplistic opera- 
tion [1]. Most pulse oximeters require a relatively small amount of hardware. Pulse oximetry is the non-invasive measurement of the oxygen saturation $\left(\mathrm{SpO}_{2}\right)$. [2] Oxygen saturation is defined as the measurement of the amount of oxygen dissolved in blood, based on the detection of Hemoglobin and Deoxyhemoglobin. Two different light wavelengths are used to measure the actual difference in the absorption spectra of $\mathrm{HbO} 2$ and $\mathrm{Hb}$. The bloodstream is affected by the concentration of $\mathrm{HbO} 2$ and $\mathrm{Hb}$, and their absorption coefficients are measured using two wavelengths $660 \mathrm{~nm}$ (red light spectra) and $940 \mathrm{~nm}$ (infrared light spectra). Deoxygenated and oxygenated hemoglobin absorb different wavelengths. Deoxygenated hemoglobin $(\mathrm{Hb})$ has a higher absorption at $660 \mathrm{~nm}$ and oxygenated hemoglobin ( $\mathrm{HbO} 2)$ has a higher absorption at $940 \mathrm{~nm}$. [3]

\section{Literature Survey}

In [1], for reducing the power consumption they used pulse oximeter adjective samples an input signal from a sensor in the absence of overriding conditions. For analysis purpose different sampling mechanisms has been deployed and they used tested individually or in combination. One of the method includes reducing the duty cycle of a drive current to a Sensor emitter, intermittently powering-down a front-end interface to a Sensor detector' or increasing the time Shift between processed data blocks. The internal parameters as well as output parameters has been monitored to trigger or override a reduced power consumption state. In such a way, a oximeter power consumption analyzed without considering high noise conditions or oxygen desaturations.

Similarly, in paper [2], recent invention regarding oximeter has been significant improvised and provides a multifunction's. The number of improvements that could be incorporated into a pulse oximeter probe to detect when a probe has become repositioned from a patient and/or to prevent a probe-off condition. A probe-off condition occurs when the optical probe becomes partially or completely repositioned from the patient, but continues to detect an AC signal within the operating region of the pulse oximeter. In one aspect, the present invention provides electrical contacts that contact with the skin of a patient when the probe is properly connected. In another aspect, the present invention provides a number of louvers placed in front of the sensor's photo detector filter out oblique light rays that do not originate from a point in front of the detector. Accordingly, if the emitter and photo detector are not properly aligned, the photo detector will not produce a signal within the valid operating range of the pulse oximeter. In accordance With a method of the present invention the pulse oximeter can sound an alarm or display a warning which determines that the probe is not properly attached to the patient. Pulse oximeter has been most recommended for general anesthetic.

Pulse oximetry measures arterial oxygen saturation and was introduced into the clinical field since the 1980s. It provides a simple, portable, non-invasive and inexpensive way for monitoring blood oxygen saturation. [4] Previous studies demonstrated that most hospital staff had limited knowledge on the correct use of pulse oximetry 
and what might affect the readings. This study aimed at evaluating the extent of knowledge in pulse oximetry among the clinical staff of an emergency department. Method: A 25-item multiple-choice type questionnaire was given to the medical and nursing staff of the Accident and Emergency Department (AED) of the Alice Ho Miu Ling Nether sole Hospital. [30] In addition to demographic information, respondents were required to answer questions relating to the basic principles of pulse oximetry, recognition of physiological factors limiting its accuracy and management regarding hypothetical scenarios. The scores were expressed and analyzed on a scale of 0-100. Results: A total of 44 questionnaires were completed by 27 nurses and 17 doctors with their test scores ranging from 20 to 72 (median=46). Doctors (median=56) did better than nurses (median=44) in general $(\mathrm{P}=0.036)$ but this advantage, however, did not apply to questions on hypothetical scenarios where nurses performed better on the contrary.[5] Correlation analysis proved that there was no linear relationship between respondents' test scores and their years of clinical experience ( $\mathrm{r}=0.051$, Spearman). This absence of linear relationship also applied to individual doctors $(r=0.244$, Spearman) and nurses groups ( $\mathrm{r}=0.162$, Spearman).

\section{Pulse oximeter implementation}

The pulse oximeter is implemented using the Freescale MCU Kinetis K53 which embeds the following key features for the pulse oximetry signal treatment, among other medical oriented applications:

- 32-bit ARM ${ }^{\circledR}$ Cortex $^{\mathrm{TM}}-\mathrm{M} 4$ core up to $100 \mathrm{MHz}$, bus speed up to $50 \mathrm{MHz}$

- DSP instructions for signal filtering

- Two Operational Amplifiers (OpAmp)

- Two Trans impedance Amplifiers (TRIAMP)

- USB connectivity as host, device or On-The-Go (OTG)

- Up to four pairs of differential and 24 single-ended 16-bit ADC channels

- 3 x 16-bit Flex Timer Modules (FTM) with PWM capability

\subsection{Functional description}

The measurement starts when the MCU generates a PWM signal that varies the LED intensity. Because every person has a different finger size and skin color, the LED needs to be calibrated to acquire an accurate signal. LED calibration is performed by taking the LED filtered baseline and using an algorithm described in the Software model which changes the PWM duty cycle value to adjust the LED intensity for every kind of user. [6] The LED driver circuit helps to drive LEDs so that power is not provided directly by the MCU. Using transistors, the LEDs are powered directly by the VCC line and controlled by the MCU. The switch control pin on the MCU selects which LED is turned on at that time. Light from Red and I Red LEDs on the sensor travels through the finger and the non-absorbed light is received in the photodetector. [7] 
The signal passes through a current to the voltage converter where it is filtered, amplified, and converted into a voltage. The signal is now multiplexed to its respective filter and amplification stage, depending on whether it is Red or IRed LED. [22] At this stage, the signal is treated and most of the noise is removed. The signal is also amplified in order to be detected easily by the MCU ADC. The filtered signal is then sent to an ADC channel on the MCU. One sample of the Red filtered signal, Red baseline, IRed filtered signal, and IRed baseline are taken every $1 \mathrm{~ms}$. Samples are captured using the embedded 16-bit ADCs and filtered using a $0.5 \mathrm{~Hz}$ to $150 \mathrm{~Hz}$ FIR (Finite Impulse Response) software filter on the Kinetis K53 MCU for high frequency and DC component removal, taking advantage of the MAC (Multiply and Accumulate) DSP instruction. [8] Samples are stored on a software buffer and averaged. A peak detection algorithm is used to determinate the AC component of the signal that is generated by the pulsatile arterial blood absorption. This is the part of the signal which is used for $\mathrm{SpO} 2$ and beats per minute (bpm) calculation. The samples taken and the calculated data (SpO2 and bpm) are sent to a GUI on a computer. More information about the software process can be found in Software model. [9]

\section{Proposed System Overview}

\section{1 $\mathrm{SpO}_{2}$ sensor}

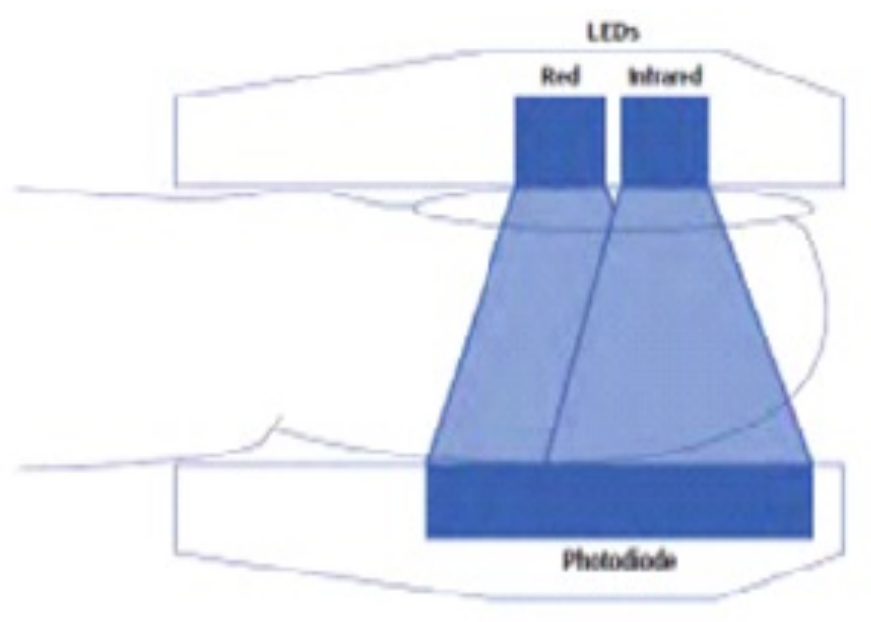

Fig. 1. $\mathrm{SpO}_{2}$ Sensor

The SPO2 is stands for, Saturation of Hemoglobin with Oxygen as Measured by Pulse Oximetry. SPO2 is defined as a ratio of the level oxygenated hemoglobin over the total hemoglobin level. In pulse oximeter there is two LEDS; one is Red LED and another Infrared LED. [1] The pulse oximeter measure oxygen saturation on the basis of BEER LAMBERT LAW. 
The BEER LAMBERT Law. This law states that the linear relationship between absorbance and concentration of species. When the light pass through the finger then on the basis of light absorbance and concentration of species we calculate ratio by BEER LAMBERT LAW and finally get the value of oxygen saturation and heart rate of patient. The PULSE OXIMETER never works without beer lambert law.SPO2 range in between 95-100.

Oxygen saturation $=\mathrm{hbo} 2 /$ total hemoglobin.

The heart rate is determined by measuring the elapsed time between peaks of the IR signal.

Heart rate $=60 /$ periods $(\mathrm{sec})$.

\subsection{Pulse Oximeter MSP432 Microcontroller}

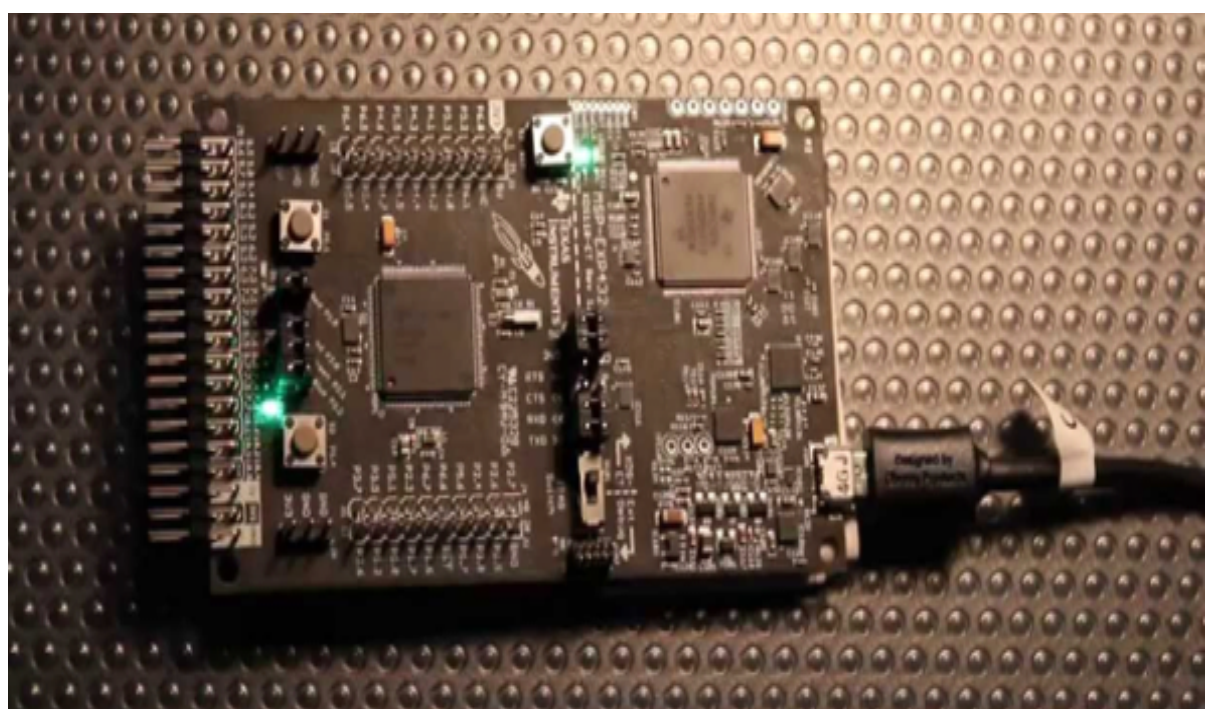

Fig. 2. MSP432 Microcontroller

The MSP432 Microcontroller is an ultra low power microcontroller; it is very widely used in Pulse Oximeter applications. It is the ARM CORTEX M4 series. It has $32 \mathrm{~KB}$ ROM and $64 \mathrm{~KB}$ SROM memory also have $256 \mathrm{~KB}$ flush memory. Up to Four 16-Bit Timers, Each With up to Five Capture, Compare, PWM Capability and Two 32-Bit Timers, Each with Interrupt Generation Capability It is an 80 pin Microcontroller consists of $16 / 32$ bit wide timers. [11] 


\subsection{6x2 LCD display-}

LCD (Liquid Crystal Display) screen is an electronic display module and find a wide range of applications. A $16 \times 2$ LCD display is very basic module and is very commonly used in various devices and circuits. [12] A $16 \times 2$ LCD means it can display 16 characters per line and there are 2 such lines. In this LCD each character is displayed in 5x7 pixel matrix. This LCD has two registers, namely, Command and Data.

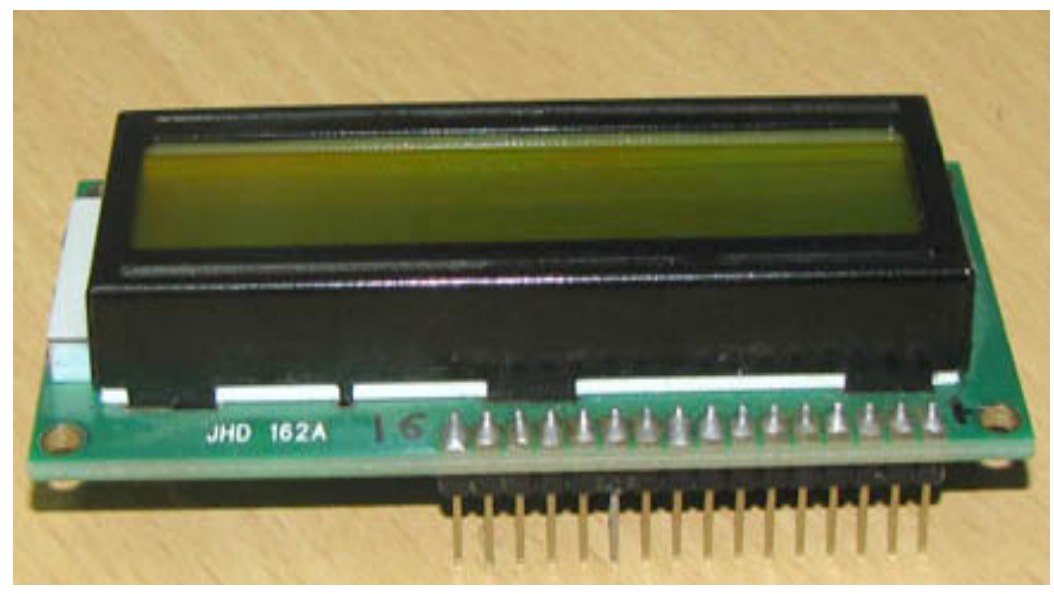

Fig. 3. Liquid Crystal Display

\section{$5 \quad$ Methodology}

\subsection{System Architecture}

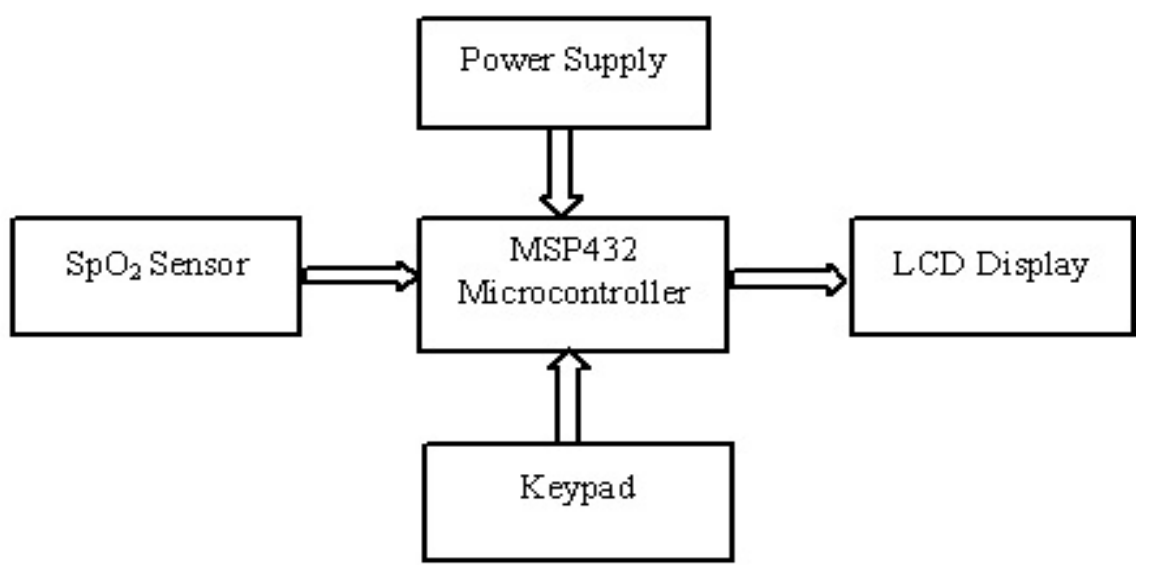

Fig. 4. Block Diagram 


\subsection{Overview}

Sensor Module. SPO2 sensors DB9 pin have connection like 2, 3 no pins connected to R and IR sensor. 5, 9 no pins connected to photodiode. The sensor emits biophysical signal on finger and the sensor send the red and infrared led absorbance data to microcontroller. [13]

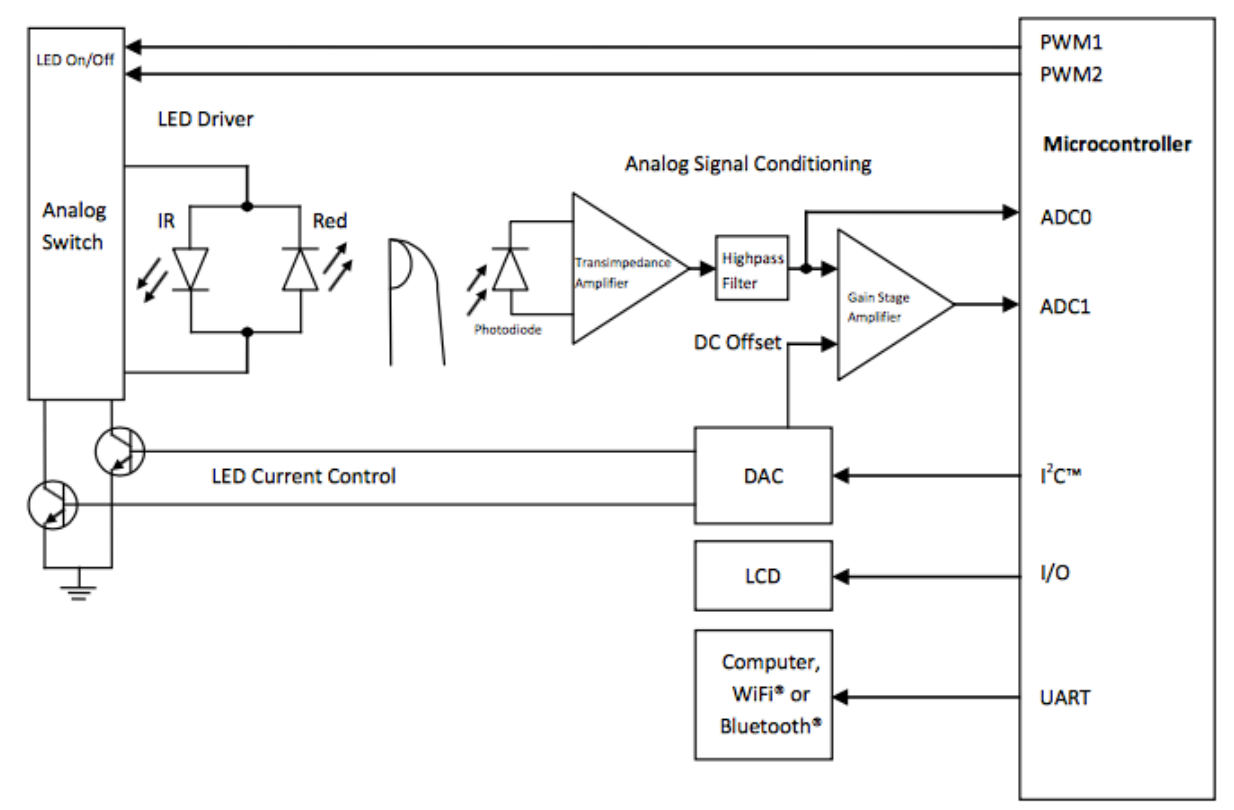

Fig. 5. System Architecture

Data Processing Module. The data processing module contains a MSP432 pulse oximeter board used for processing the signal to calculate oxygen saturation and heart rate. The incoming signals are amplified by using internal op-amps and Microcontroller unit separates the infra-red and red components and apply beer lambert law on basis of absorbance and concentration calculate ratio. [14] The proposed pulse oximeter consists of pulse oximeter MSP432 board connected to a MSPFET430UIF JTAG Debugger for debugging process.[15] The system requires 5v DC power supply .According to the Beer-Lambert law (or Beer's law) which states that there is a linear relationship between absorbance and concentration of an absorbing species. In general Beer-Lambert law statement is usually written as:

$$
\mathrm{A}=\mathrm{a}(\lambda) * \mathrm{~b} * \mathrm{c}
$$

Where $\mathrm{A}$ is defined as the measured absorbance, a $(\lambda)$ is a wavelength-dependent absorptivity coefficient, $\mathrm{b}$ is the path length, and $\mathbf{c}$ is the analyze concentration. When working in concentration units of molarity, the Beer-Lambert law is written as:

$$
\mathrm{A}=\varepsilon * \mathrm{~b} * \mathrm{c}
$$


Where $\Xi$ is the wavelength-dependent molar absorptivity coefficient with units of $\mathrm{M}^{-1} \mathrm{~cm}^{-1}$. Data are frequently reported in percent transmission $\left(\mathrm{I} / \mathrm{I}_{0} * 100\right)$ or in absorbance $\left[\mathrm{A}=\log \left(\mathrm{I} / \mathrm{I}_{0}\right)\right]$.

Naturally transmittance is directly related to absorbance. Above mentioned Transmittance Equation may be further extended by taking the natural log to give what is called the un-scattered absorbance $(A)$. Thus, the absorbance in the system of equations can only be found by measuring received intensities and comparing them to the original intensities of transmitted light. There are several problems here. [16] First, the intensity of the original incident light is very difficult to determine accurately. LEDs from the factory do not all emit the same intensity. Further, LEDs are not monochromatic; their intensity is spread over a spectrum of wavelengths. The need to know the value of $I 0$ is eliminated by taking advantage of the pulsating properties of arteries. For development of pulse oximeter consist MSP432 board connected to sensors and LCD. [17] The board is full set resources inbuilt which consist of ADC, DAC, UART, DMA and a liquid crystal display (LCD).

Display Module. The system having 16x2 LCD display module. The output of SPO2 sensor and MSP432 microcontroller is display on LCD. Keypad, LCD, SPO2 sensor connected to the MSP430FG439 microcontroller.

Naturally transmittance is directly related to absorbance. Above mentioned Transmittance Equation may be further extended by taking the natural log to give what is called the un-scattered absorbance $(A)$. Thus, the absorbance in the system of equations can only be found by measuring received intensities and comparing them to the original intensities of transmitted light [23]. There are several problems here. First, the intensity of the original incident light is very difficult to determine accurately. [18] LEDs from the factory do not all emit the same intensity. Further, LEDs are not monochromatic; their intensity is spread over a spectrum of wavelengths. The need to know the value of $I 0$ is eliminated by taking advantage of the pulsating properties of arteries.[26] For development of pulse oximeter consist MSP432 board connected to sensors and LCD. The board is full set resources inbuilt which consist of ADC, DAC, UART, DMA and a liquid crystal display (LCD).[19]

\section{Applications}

This section provides about a review of applications of wearable and ambient sensors and systems that are relevant to the field of rehabilitation. The material is organized in five sub-sections devoted to summarizing

Applications focused on:

1. Health and wellness monitoring,

2. Safety monitoring,

3. Home rehabilitation,

4. Assessment of treatment efficacy, and

5. Early detection of disorders. 


\section{Conclusion}

In this project we used Beer's lambert law to get oxygen level and this will show on LCD. This will used two wavelength of light like $990 \mathrm{~nm}$ for red light and $660 \mathrm{~nm}$ for infrared light. Two wavelengths are used because the absorptivity of oxy hemoglobin and reduced hemoglobin differ.

\section{Future Scope}

In future, heart rate and respiration rate [8] can also be measured by pulse Oximeter along with $\mathrm{SpO} 2$ providing further functionality to the project. Also, different sensors like blood pressure sensor and ECG sensor can be added to measure other parameters as well. Further, by integrating GSM with our project, we can send notification messages to a medical expert helping in immediate alert in case of emergencies. Provision of information regarding prescription and reminder for medicine intake can also be included. [20]

\section{References}

[1] Kiani, Massi E., and Mohamed K. Diab. "Pulse oximeter probe-off detection system." U.S. Patent No. 6,526,300. 25 Feb. 2003.

[2] Amoore, J. N. Pulse Oximetry: An Equipment Management Perspective. IEEE. Pulse Oximetry: A Critical Appraisal, IEE Colloquium on 29 May 1996. pg. 6/1 6/7.

[3] Webster, J. G., ed. Design of Pulse Oximeters. Institute of Physics Publishing. Bristol. 1997. https://doi.org/10.1887/0750304677

[4] Gupta, R. C., et al. Design and Development of Pulse Oximeter.IEEE Proceedings: $14^{\text {th }}$ Conference Biomedical Engineering Society of India. 15-18. February 1996. pg. 1/13 1/16.

[5] O Reilly, G., Tuohy, G. Methods of Assessment of Pulse Oximeters. IEEE. Pulse Oximetry: A Critical Appraisal, IEE Colloquium on 29 May 1996. pg. 6/1 6/7.

[6] Webster, J. G., ed. Medical Instrumentation: Application and Design. 3 rd ed. John Wiley \& Sons, Inc. New York. 1998. pg. 452

[7] J. G.Webster, Design of Pulse Oximeters. Bristol, U.K.: Inst. Phys..,1997. https://doi.org/10.1887/0750304677

[8] PalmSAT® 2500 Digital Handheld Pulse Oximeter User Guide. Minneapolis, MN: Nonin Medical, 2004.

[9] Pedro Magana-Espinoza, Raul Aquino-Santos, Nestor Cardenas-Benitez2, Jose AguilarVelasco, Cesar Buenrostro-Segura, Arthur Edwards-Block and Aldo Medina-Cass , 2015 "WiSPH: A Wireless Sensor Network-Based Home Care Monitoring System".

[10] Muneer Bani Yassein, Mohammad Hamdan, Hisham Shehadeh and Lina Mrayan, 2015 "An Improvement on Health Monitoring System for Multiple Patients Using Wireless Sensor Network".

[11] Neeta Desai, Saniya Ansari, 2015 "Wireless Reliable Embedded System for Patient Monitoring".

[12] P.Karthick, C.Sureshkumar, P.Arunprasa, S.Pusparaj, M.Jagadeeshraja, N.Suthanthira Vanitha, 2014 "Embedded Based Real-time Patient Monitoring System".

[13] Khalifa AlSharqi, Abdelrahim Abdelbari, Ali Abou-Elnour, and Mohammed Tarique, 2014,"zigbee based wearable remote healthcare monitoring system for elderly patients". 
[14] Xiaonan Wang, Deguang Le, Hongbin Cheng, Conghua Xie, 2014 “All-IP wireless sensor networks for real-time patient monitoring".

[15] Anju.C.K, Lekshmi.S, 2014 "Wireless Biomedical Parameter Monitoring System Using Arm Microcontroller: A Review".

[16] K. Navya, Dr. M. B. R. Murthy, 2013 “A Zigbee Based Patient Health Monitoring System"

[17] Rajasekaran.S, Kumaran.P, Premnath.G, Karthik.M, 2013 "Human health monitoring using wireless sensors network (WSN)".

[18] A. AL-Marakeby, 2013 "Camera-Based Wireless Sensor Networks for E-Health “.

[19] Elham Shahinfard, Maher Sid-Ahmed, Majid Ahmadi ,2007" An Improved Motion Adaptive Deinterlacing Method Using Variable Block-Size Motion Detection".

[20] [20]. Johannes Barnickel, Hakan Karahan, Ulrike Meyer, "Security and Privacy for Mobile Electronic Health Monitoring and Recording Systems" IEEE 2010.

[21] Juan M. Corchado, Javier Bajo, Dante I. Tapia, and Ajith Abraham, "Using Heterogeneous Wireless Sensor Networks in a Telemonitoring System for Healthcare," IEEE Transactions on Information Technology in Biomedicine, Vol. 14, No. 2, March 2010, pp.234-240. https://doi.org/10.1109/TITB.2009.2034369

[22] Luan Ibraimi, Muhammad Asim, Milan Petko vic, "Secure Management of Personal Health Records by Applying Attribute-Based Encryption,” IEEE 2010.

[23] M. Li and W. Lou," Data Security and Privacy in Wireless Body Area networks," IEEE Wireless Comm., Feb. 2010, pp. 51-58.

[24] Qian Wang and Kui Ren Wenjing Lou Yanchao Zhang, "Dependable and Secure Sensor Data Storage with Dynamic Integrity Assurance", IEEE INFOCOM 2009, pp.954-962. https://doi.org/10.1109/INFCOM.2009.5062006

[25] R. Suji Pramila, A. Shajin Nargunam, "A Study On Data Confidentiality In early Detection Of Alzheimer's Disease,” IEEE 2012, pp.1004-1008.

[26] Reza S. Dilmaghani, Hossein Bobarshad, M. Ghavami, Sabrieh Choobkar, and Charles Wolfe, "Wireless Sensor Networks for Monitoring Physiological Signals of Multiple Patients," IEEE Transactions on biomedical circuits and systems, vol. 5, no. 4, august 2011, pp.347-356. https://doi.org/10.1109/TBCAS.2011.2114661

[27] Rong Fan, Ling-Di Ping, Jian-Qing Fu, Xue-Zeng Pan, "The New Secure and Efficient Data Storage Approaches for Wireless Body Area Networks," IEEE 2010. https://doi.org/10.1109/WCSP.2010.5633690

[28] Yifeng He, Wenwu Zhu and Ling Guan, "Optimal Resource Allocation for Pervasive Health Monitoring Systems with Body Sensor Networks", IEEE Transactions on Mobile Computing, Vol.10, No.11, November 2011, pp.1558-1575. https://oi.org/10.1109/ $\underline{\text { TMC.2011.83 }}$

\section{Authors}

Ms. Shruti Daddikar is with Dr. D.Y.Patil School of Engineering and Technology, Savitribai Phule Pune University, Pune, Maharashatra, India (Shruti.daddikar@gmail.com).

Dr. Roshani Ade is with Dr. D.Y.Patil School of Engineering and Technology, Savitribai Phule Pune University, Pune, Maharashatra, India.

Article submitted 06 January 2017. Published as resubmitted by the authors 23 March 2017. 